\title{
Quantum-state-preserving optical pulse reshaping and multiplexing by four-wave mixing in a fiber
}

McKinstrie, C. J. ; Andersen, Lasse Mejling; Rottwitt, Karsten; Raymer, M. G.

Published in:

CLEO Technical Digest

Publication date:

2012

Document Version

Publisher's PDF, also known as Version of record

Link back to DTU Orbit

Citation $(A P A)$ :

McKinstrie, C. J., Andersen, L. M., Rottwitt, K., \& Raymer, M. G. (2012). Quantum-state-preserving optical pulse reshaping and multiplexing by four-wave mixing in a fiber. In CLEO Technical Digest (pp. JW4A.117). Optical Society of America.

\section{General rights}

Copyright and moral rights for the publications made accessible in the public portal are retained by the authors and/or other copyright owners and it is a condition of accessing publications that users recognise and abide by the legal requirements associated with these rights.

- Users may download and print one copy of any publication from the public portal for the purpose of private study or research.

- You may not further distribute the material or use it for any profit-making activity or commercial gain

- You may freely distribute the URL identifying the publication in the public portal 


\title{
Quantum-state-preserving optical pulse reshaping and multiplexing by four-wave mixing in a fiber
}

\author{
C. J. McKinstrie, ${ }^{1}$ L. Mejling, ${ }^{2}$ M. G. Raymer $^{3}$ and K. Rottwitt ${ }^{2}$ \\ ${ }^{1}$ Bell Laboratories, Alcatel-Lucent, Holmdel, New Jersey 07733 \\ ${ }^{2}$ Department of Photonics, Technical University of Denmark, 2800 Kongens Lyngby, Denmark \\ ${ }^{3}$ Department of Physics, University of Oregon, Eugene, Oregon 97403
}

\begin{abstract}
Nondegenerate four-wave mixing driven by two pulsed pumps transfers the quantum state of an input signal pulse to an output idler pulse, which is a frequency-translated and reshaped version of the signal. By varying the pump shapes appropriately, one can connect signal and idler pulses with arbitrary durations and shapes. This process enables a variety of functions required by quantum information networks.
\end{abstract}

(C) 2011 Optical Society of America

OCIS codes: 190.4380 nonlinear optics, four-wave mixing; 270.5565 quantum communications.

\section{Summary}

A key requirement of quantum information networks is the ability to transport quantum states between nodes [1,2]. These nodes (material-based quantum memories with resonance wavelengths in the range 300-800 nm) could be nearby (in the same device) or distant (linked by optical fibers with low-loss windows centered on 1310 or $1550 \mathrm{~nm}$ ). Their resonance wavelengths could be the same, or similar, or could differ significantly. Furthermore, the durations and shapes of pulses emitted by quantum memories differ significantly from the short bell-shaped pulses favored by communication systems. To optimize the transmission of quantum information, and the exchange of information between different channels, the ability to frequency translate (FT) and reshape optical pulses, while preserving their quantum states, is essential.

Quantum-state-preserving FT is enabled by three-wave mixing (TWM) in a crystal $[1,3]$. In this process, a strong pump wave $(p)$ couples weak signal $(s)$ and idler $(r)$ waves $\left(\pi_{s} \longleftrightarrow \pi_{p}+\pi_{r}\right.$, where $\pi_{j}$ represents a photon with frequency $\omega_{j}$ ). Recently, it was shown theoretically that TWM, in conjunction with spectral phase modulation and propagation [4], or complicated dispersion engineering [5], can connect pulses with disparate durations.

Quantum-state-preserving FT is also enabled by four-wave mixing (FWM) in a fiber $[6,7]$. In the nondegenerate FWM process called Bragg scattering (BS), two pumps ( $p$ and $q$ ) couple the signal and idler $\left(\pi_{s}+\pi_{p} \longleftrightarrow \pi_{q}+\pi_{r}\right)$. BS has been used in conventional communication experiments to provide tunable FT and multiplexing. It also has the ability to reshape pulses arbitrarily, without additional processing or complicated dispersion engineering.

$\mathrm{BS}$ is governed by the coupled-mode equations (CMEs)

$$
\left(\partial_{z}+\beta_{r} \partial_{t}\right) a_{r}(z, t)=i \gamma_{p q}(z, t) a_{s}(z, t),\left(\partial_{z}+\beta_{s} \partial_{t}\right) a_{s}(z, t)=i \gamma_{p q}^{*}(z, t) a_{r}(z, t),
$$

where $a_{r}$ and $a_{s}$ are the annihilation operators of the idler and signal, respectively, $\beta_{r}$ and $\beta_{s}$ are the group slownesses, $\gamma_{p q}(t, z)=\gamma A_{p}\left(t-\beta_{s} z\right) A_{q}^{*}\left(t-\beta_{r} z\right)$ is the pump-induced coupling function, $\gamma$ is proportional to the Kerr coefficient and $A_{j}$ is a pump amplitude [6]. Pump $p$ co-propagates with the signal, whereas pump $q$ co-propagates with the idler [7]. We determined the Green functions associated with the CMEs analytically, and performed their Schmidt decompositions numerically. The Schmidt modes (temporal eigenfunctions) are the optimal input and output pulse shapes, and are related to the pump shapes. The Schmidt coefficients (eigenvalues) are intermodal conversion efficiencies.

The signal-to-idler Green function $G_{r s}$ was decomposed numerically for a fiber that was long enough to allow a complete pump-signal interaction. In Fig. 1(a), the first and second Schmidt coefficients $\left(\left|\rho_{1}\right|^{2}\right.$ and $\left.\left|\rho_{2}\right|^{2}\right)$ are plotted as functions of the interaction strength $\left[\bar{\gamma}=\gamma /\left(\beta_{r}-\beta_{s}\right)\right]$. In the low-strength regime, the first coefficient (conversion efficiency) $\left|\rho_{1}\right|^{2} \approx \bar{\gamma}^{2}$. In the high-strength regime, $\left|\rho_{1}\right|^{2}$ tends monotonically to 1 as $\bar{\gamma}$ increases, so complete conversion is possible. For efficiencies as high as 0.70 , the value of the second Schmidt coefficient is no larger than 0.01, so the Green function is nearly separable: The first (target) Schmidt mode is converted with reasonable efficiency, whereas the other modes are unaffected. For higher efficiencies, the second coefficient increases rapidly and the potential exists 

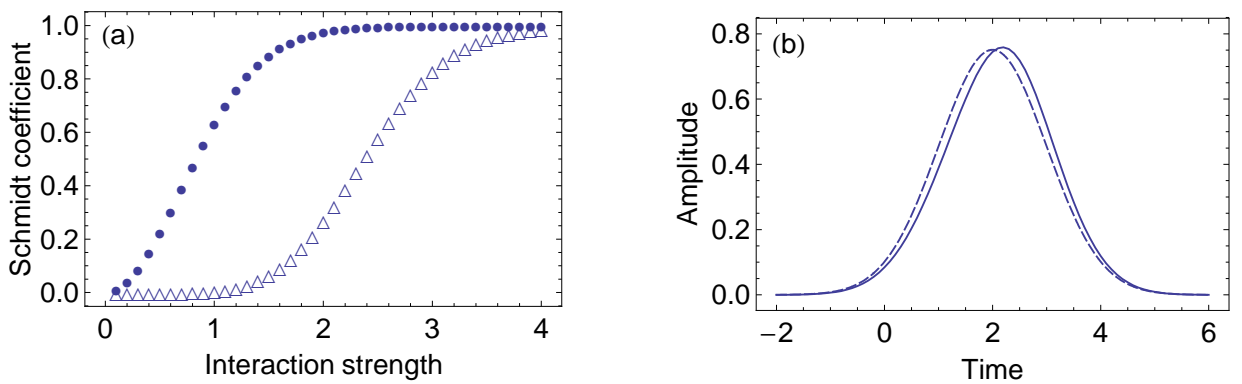

Fig. 1. (a) Schmidt coefficients $\left(\left|\rho_{n}\right|^{2}\right)$ plotted as functions of the strength parameter $(\bar{\gamma})$. The solid circles denote $\left|\rho_{1}\right|^{2}$ and the hollow triangles denote $\left|\rho_{2}\right|^{2}$. (b) Lowest-order Schmidt mode (solid curve) and (common) pump shape (dashed curve) plotted as functions of time $(t / \tau)$ for the strength parameter $\bar{\gamma}=0.834$.

for intermodal crosstalk. The first Schmidt mode of $G_{r s}$ is displayed in Fig. 1(b) for the case in which $\bar{\gamma}=0.834$. This strength parameter corresponds to a conversion efficiency of 50\%, which is suitable for entanglement generation or Hong-Ou-Mandel (HOM) interference. The pumps are identical Gaussians with root-mean-square duration $\tau$ and the (common) Schmidt mode differs only slightly from a Gaussian, as predicted by theory.

The first pair of Schmidt modes of $G_{r s}$ is displayed in Fig. 2 for the case in which $\bar{\gamma}=1.544$. This strength parameter corresponds to a conversion efficiency of $90 \%$, which is high enough for reliable quantum FT or entanglement swapping. Pumps $p$ and $q$ are zeroth- and first-order Hermite-Gauss functions, respectively, so the wavepacket is reshaped. The Schmidt modes are distorted Hermite-Gauss functions, as predicted by theory.
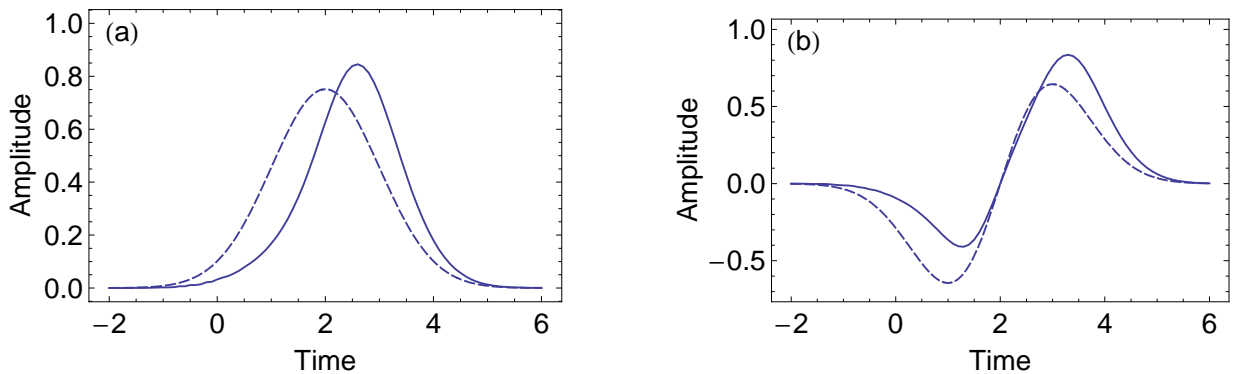

Fig. 2. Lowest-order (a) signal and (b) idler Schmidt modes (solid curves) and pump shapes (dashed curves) plotted as functions of time $(t / \tau)$ for the strength parameter $\bar{\gamma}=1.544$.

In summary, BS driven by pulsed pumps was studied in detail. The Green functions and Schmidt decompositions associated with this FT process were determined for arbitrary conversion efficiencies. BS enables the generation of entanglement, or HOM interference, between photons with different carrier frequencies and wavepackets, and photon FT, or entanglement swapping, with simultaneous wavepacket reshaping that is limited only by the available pump shapes (waveforms). Since arbitrary pump-waveform generation is possible [8], the future of FT by BS looks bright.

\section{References}

1. S. Tanzilli et al., Nature (London) 437, 116 (2005).

2. H. J. Kimble, Nature (London) 453, 1023 (2008).

3. J. Huang and P. Kumar, Phys. Rev. Lett. 68, 2153 (1992).

4. D. Kielpinski et al., Phys. Rev. Lett. 106, 130501 (2011).

5. B. Brecht et al., New J. Phys. 13, 065029 (2011).

6. C. J. McKinstrie et al., Opt. Express 13, 9131 (2005).

7. H. J. McGuinness et al., Phys. Rev. Lett. 105, 093604 (2010).

8. S. J. Cundiff and A. M. Weiner, Nat. Photon. 4, 760 (2010). 